\title{
Mortalidad en personas con síndrome de fatiga crónica
}

\author{
Mortality in people with chronic fatigue syndrome
}

Roberts E. Lancet 2016;387:1638-43.

\section{Objetivo}

Evaluar las tasas de mortalidad por todas las causas, por suicidio y por cáncer en pacientes con diagnóstico de síndrome de fatiga crónica (SFC) atendidos en centros de segundo y tercer nivel de complejidad.

\section{Diseño y lugar}

Estudio de cohortes, retrospectivo; realizado en Inglaterra y Gales.

\section{Pacientes}

Los datos fueron obtenidos del Registro Clínico de Búsqueda Interactiva (en inglés: Clinical Record Interactive Search Register - CRIS) de la South London and Maudsley NHS Foundation Trust, también conocida como SLaM. Esta fundación del sistema de salud del Reino Unido, se especializa en salud mental y comprende tres hospitales psiquiátricos, la Unidad Ladywell del Hospital Universitario Lewisham y más de 100 sitios comunitarios y 300 equipos clínicos. Los participantes fueron incluidos en el estudio si habían tenido contacto con el servicio de fatiga crónica y habían recibido el diagnóstico de SFC.

\section{Medición de resultados principales}

Se calcularon las tasas de mortalidad por todas las causas, por suicidio y por cáncer asentadas en el registro de la SLaM durante un período de siete años y se compararon con estadísticas de mortalidad según sexo y edad de Inglaterra y Gales.

\section{Resultados}

Entre el 1 de enero de 2007 y el 31 de diciembre de 2013 se identificaron 2.147 casos de SFC (1.533 mujeres y 614 hombres), y 17 muertes (11 mujeres y 6 varones). No hubo diferencia en los índices de mortalidad estandarizados por edad y por sexo (standardised mortality ratios - SMR) por todas las causas (SMR 1,14; IC 95\% 0,65 a 1,85; p $=0,67)$ o mortalidad específica por cáncer $(1,39 ; 0,60$ a 2,73; $p=0,45)$ en pacientes con SFC al compararlos con la población general de Inglaterra y Gales. En cambio sí hubo un aumento significativo en la mortalidad específica por suicidio (SMR 6,85; 2,22 a 15,98; $p=0,002$ ).

\section{Conclusiones}

Los autores concluyen que no se observó un aumento de muertes por todas las causas ni por cáncer en los pacientes con SFC pero sí un aumento de la mortalidad por suicidio. En esta línea, destacan la necesidad de que los médicos sean conscientes de este dato para evaluar el riesgo de suicidio de manera adecuada en los pacientes con SFC.

Fuente de financiamiento: National Institute for Health Research (NIHR) Biomedical Research Centre at South London and Maudsley NHS Foundation Trust and King's College London.

\section{Comentario}

El síndrome de fatiga crónica (SFC) es descripto como la instalación súbita de fatiga (generalmente tras un cuadro gripal o seudogripal) que persiste por más de seis meses y se asocia con síntomas musculoesqueléticos y neuropsicológicos. Los criterios para su diagnóstico son los siguientes: 1) fatiga persistente o recurrente, clínicamente inexplica$\mathrm{da}$, de comienzo reciente, que no se relaciona con la actividad física ni cede con el reposo y genera una reducción significativa en el rendimiento laboral, educativo, social o personal y 2) cuatro o más de los siguientes síntomas durante por lo menos seis meses consecutivos y que no estaban presentes antes del comienzo de la fatiga: dificultad en la concentración o en la memoria a corto plazo, odinofagia, adenopatías dolorosas en las cadenas cervicales o axilares, mialgias, poliartralgias sin artritis, cefalea de instalación nueva o con un patrón o severidad nuevos, sueño no reparador (falta de mejoría de los síntomas después de dormir), decaimiento posterior al ejercicio de más de un día de duración'. Su diagnóstico se realiza por exclusión, de acuerdo a los criterios mencionados, en pacientes con examen físico y estudios complementarios normales. El estudio presentado tiene varias limitaciones, algunas de las cuales reconocen los mismos autores: 1) la población evaluada correspondía a pacientes con diagnóstico de SFC realizado en instituciones de segundo o tercer nivel de atención especializadas en salud mental y que ofrecían un servicio especializado para dicho problema de salud, por lo que los resultados no necesariamente son aplicables a la población general; 2) entre los pacientes con SFC hubo más suicidios y los autores concluyen sobre la importancia de estar alerta a este dato, sin embargo reconocen que si hubiera habido dos muertes menos por suicidio en el grupo con diagnóstico de SFC el aumento de riesgo no sería estadísticamente significativo; 3) la observación de la cohorte fue por solo siete años y el SFC es, como su nombre lo destaca, crónico por lo que no se puede descartar que, con el seguimiento por más años, los resultados fueran diferentes; 4) no se evaluaron otras variables como el tabaquismo, el índice de masa corporal o la presencia de otras enfermedades crónicas que podrían afectar el riesgo de mortalidad; 5) se utilizaron datos provenientes de una base de salud mental, hecho que podría sesgar la muestra (no todas las personas acceden a consultar a una institución psiquiátrica por vergüenza, temor, resistencia, etc. o la población evaluada podría tener cuadros más graves o mayor comorbilidad psiquiátrica que la población general) y 6) en el intento de tener una buena muestra se utilizaron diferentes criterios de diagnóstico de SFC para incluir pacientes: criterios de Oxford ${ }^{2,3}$ (los cumplía el 65\% de los pacientes), criterios de definición de casos de $1994^{4}$ (58\% de los pacientes) y criterios de $\mathrm{NICE}^{5}$ (88\% de los pacientes). El problema es que los criterios de Oxford son bastante cuestionados porque no consideran a la depresión como un criterio de exclusión para diagnosticar SFC (lo que podría haber conducido a incluir pacientes con comorbilidad psiquiátrica en la muestra evaluada).

\section{Conclusiones del comentador}

Más allá de estas cuestiones, como médica debo confesar que dudo de la verdadera existencia del SFC, aunque reconozco que puede servir como un diagnóstico operativo en algunas circunstancias y con algunos pacientes. Con respecto a este trabajo en particular, pienso que, para la clínica cotidiana, es más importante lo que no halló (diferencia de mortalidad por todas las causas y por cáncer entre los pacientes sin y con SFC) que lo que sí encontró (mayor mortalidad por suicidio entre los pacientes con SFC).

Paula Carrete [ Servicio de Medicina Familiar y Comunitaria del Hospital Italiano de Buenos Aires. paula.carrete@ hospitalitaliano.org.arr ]

Carrete P. Mortalidad en personas con síndrome de fatiga crónica. Evid Act Pract Ambul 2016. 19(4):119. Comentado de: Roberts E. Mortality of people with chronic fatigue syndrome: a retrospective cohort study in England and Wales from the South London and Maudsley NHS Foundation Trust Biomedical Research Centre (SLaM BRC) Clinical Record Interactive Search (CRIS) Register. Lancet 2016;387:1638-43. PMID: 26873808.

Referencias

1. Carrete P, Rubinstein E. Cansancio. Buenos Aires: delhospital ediciones; 2015

2. Sharpe MC, Archard LC, Banatvala JE, et al. A report—chronic fatigue syndrome: guidelines for research. J R Soc Med 1991; 84: 118-21

3. Sharpe M, Chalder T, Palmer I, Wessely S. Chronic fatigue syndrome. A practical guide to assessment and management. Gen Hosp Psychiatry 1997; 19: 185-99

4. Fukuda K, Straus SE, Hickie I, Sharpe MC, Dobbins JG, Komaroff A, and the International Chronic Fatigue Syndrome Study Group. The chronic fatigue syndrome: a comprehensive approach to its defi nition and study. Ann Intem Med 1994; 121: 953-59

5. NICE Chronic fatigue syndrome/myalgic encephalomyelits (or encephalopathy): diagnosis and management of chronic fatigue syndromeME in adults and children. NICE Cinical Guideline; CG53. 2007 